\title{
Structural and optical and characterization of poly (aniline) (PANi) films obtained by spin-coating method
}

\author{
Zina Barhoumi ${ }^{1, *}$, Noureddine Amdouni ${ }^{1}$, Jean François Stumbé ${ }^{2}$ and Amalendu Pal ${ }^{3}$ \\ ${ }^{1}$ Unité Physico-Chimie Des Matériaux Solides, Département de Chimie, Faculté des Sciences de Tunis, Campus \\ Universitaire, 2092 El Manar Tunisie \\ ${ }^{2}$ Laboratoire de Photochimie et d'Ingénierie Macromoléculaires, Université de Haute Alsace, 3rue A.werner \\ 68093 Mulhouse cedex, France \\ ${ }^{3}$ Department of Chemistry, Kurukshetra University, Kurukshetra 136 119, India
}

\begin{abstract}
The structural and optical properties of the PANi films were investigated in terms of different solvent content by using X-ray diffraction (XRD), RAMAN spectroscopy, Scanning electron microscopy (SEM), Photoluminescence (PL) and optical absorption spectroscopy. The XRD results revealed that the crystallinity through films increased as the molar ratio of dimethylacetamide (DMAc and dimethylformamide (DMF) increased to 0.6:0.4, but then decreased when the molar ratio was increased from 0.6:0.4 to 0.8:0.2. It is shown that even small clusters in PANi films can explain the observed RAMAN scattering. The results of the optical experiments showed that films prepared from different molar ratio of DMAc and DMF showed good nearinfrared characteristic in the range of $800-2000 \mathrm{~nm}$. The optical band gap value from absorption coefficient data is found to be $3.57 \mathrm{eV}$. The SEM also showed changes in the morphology of PANi films.
\end{abstract}

Keywords: Conducting polymer; Spin-Coating; Raman spectroscopy and scattering; Optical properties.

\section{Introduction}

In recent years, developments of inorganicorganic hybrid materials on nanometer scale have been receiving significant attention due to a wide range of potential applications and high absorption in the visible part of the spectrum and high mobility of the charge carriers. Polyaniline (PANi) has been one of the most thoroughly investigated conducting polymers due to its environmental stability, high density of charge storage capability, and low cost and simple synthesis ${ }^{\mathbf{1 - 3}}$. These polymers have extensive applications such as anticorrosive ${ }^{\mathbf{4 - 6}}$; sensors and biosensors ${ }^{7-9}$, as actuators ${ }^{10-11}$, in advanced biomedical materials $\mathbf{1 2}$, in electronic device $^{\mathbf{1 3}}$, in transistors ${ }^{\mathbf{1 4}}$ and hole injection layers for flexible light emitting diodes ${ }^{\mathbf{1 5 , 1 6}}$. However, such industrial applications are limited for PANi because of its poor processability and intractable nature. A pdopable (i.e., electron deficient) material, PANi exhibits reversible electrochemical and physical properties controlled by its oxidation and protonation state properties that have been exploited for the development of several technological applications including optoelectronic device. PANi can be easily polymerized by direct chemical oxidation of aniline or electrochemical polymerization ${ }^{17}$.
But, in the preparation of PANi dispersions, surfactants have been rarely employed as stabilizer and micelles as reaction medium. Han et $a l$. prepared PANi nanoparticles in anionic sodium dodecylsulfate (SDS) micelles with ammonium peroxydisulfate (APS) as an oxidant. More structured PANI particles showed a higher conductivity ${ }^{18}$. Li and co-workers successfully prepared stable nano-polyaniline waterborne latex by emulsion polymerization of aniline in micellar solution of sodium dodecylbenzene sulfonate (SDBS), dodecylbenzene sulfonic acid (DBSA), or sodium dodecylsulfate (SDS) as both emulsifier and dopant. The uniform PANi-SDS/polyvinyl alcohol (PVA) composite films were prepared by casting a direct mixture of the PANi latex and PVA aqueous solution and good properties including film formability, anticorrosivity, and electrical conductivity ${ }^{\mathbf{1 9}}$. Sainz et al. obtained soluble polyaniline-multi-wall-carbon nanotube (CNT-PANi) composites. It was found that the presence of multi-wall-carbon during the polymerization of aniline induces the formation of a planar conformation of $\mathrm{PANi}^{\mathbf{2 0}}$. Studies of $\mathrm{Ma}$ and co-workers ${ }^{21}$ have suggested that the use singlestranded DNA offers better solubilization of the CNTs prior to the water-soluble nanocomposite preparation (PANi-CNTs). 
This PANi-CNTs composite in functionalized forms exhibited good enhanced conductivity, thermal stability and luminescent behaviors. Therefore, conformational changes lead to favorable interactions between emeraldine base (EB) and CNTs and are responsible for both enhancement effects and the processability of the composite. The effect of the solvent type and casting temperature on the electrical properties of plastdoped polyaniline films was investigated by Bohli et al. the films prepared from PANi in dichloroacetic acid (DCAA) after heating treatment at $298 \mathrm{~K}$ showed the highest electrical conductivities ${ }^{22}$. In all these views, we endeavored to study the structural, optical properties of polyaniline films and to cater the needs of optical materials in near future.

\section{Experimental}

\section{Materials}

Aniline monomer $\mathrm{C}_{6} \mathrm{H}_{7} \mathrm{~N}$ was purchased from Sigma-Aldrich and bi-distilled under vacuum before use. Ammonium persulfate $\left(\left(\mathrm{NH}_{4}\right)_{2} \mathrm{~S}_{2} \mathrm{O}_{8}\right.$, APS $)$ was obtained from Fluka. N, N- dimethylacetamide and dimethylformamide were obtained from Sigma Aldrich. The chemicals were used as received without further purification.

\section{Preparation of PANi: DMAc/DMF} composites film-forming solution

Polyaniline emeraldine base (PANi-EB), which was synthesized was dissolved into variable ratio DMAc/DMF mixtures to prepare a PANi (3 wt \%) solution. Stock solutions were stirred using a magnetic stirrer for $1 \mathrm{~h}$. A Speed line Technologies P-6000 spin coater was used to spin cast polymer solutions for $30 \mathrm{~s}$ onto the substrates. All films were held under vacuum overnight prior to measurements to remove any residual solvents. The dried polymer films, in which thickness values around $100 \mathrm{~nm}$, were analyzed.

\section{Characterization}

The XRD spectra of the PANi films were measured on X'Pert PRO Alpha-1-ray diffractometer with $\mathrm{CuK} \alpha(1.5405 \AA$ Á), and it was operated at $40 \mathrm{KV}$ voltage and $50 \mathrm{~mA}$ anode current. The XRD patterns were recorded in the range of $3-65^{\circ}$ with a scan speed $0.5 \% \mathrm{~min}$. Raman spectra of films were carried out at room temperature in the range of $100-2000 \mathrm{~cm}^{-}$ 1 using FT-Raman Bruker SENTERRA which is having Nd: YAG laser source (488nm). The Photoluminescence (excitation and emission) spectra of the PANi films were recorded on Cary Eclipse spectrophoto-fluorometer using the corresponding excitation wavelength with $2.5 \mathrm{~nm}$ slits. Optical properties of the samples have been analyzed using a VARIAN spectrophotometer at room temperature over a large spectral range. The wavelength range for both the reflectance and transmittance measurements was ranged between 300-2200 nm. Scanning electron microscopy experiments were made with Philips Field Effect Gun XL-30, at an accelerating voltage of $5 \mathrm{kV}$. Image treatments were performed using the digital instructions software.

\section{Results and discussion}

\section{X-ray diffraction}

Structural properties of PANi films were characterized by $\mathrm{X}$ - ray diffraction (XRD). XRD pattern of different films is shown in Figure 1.

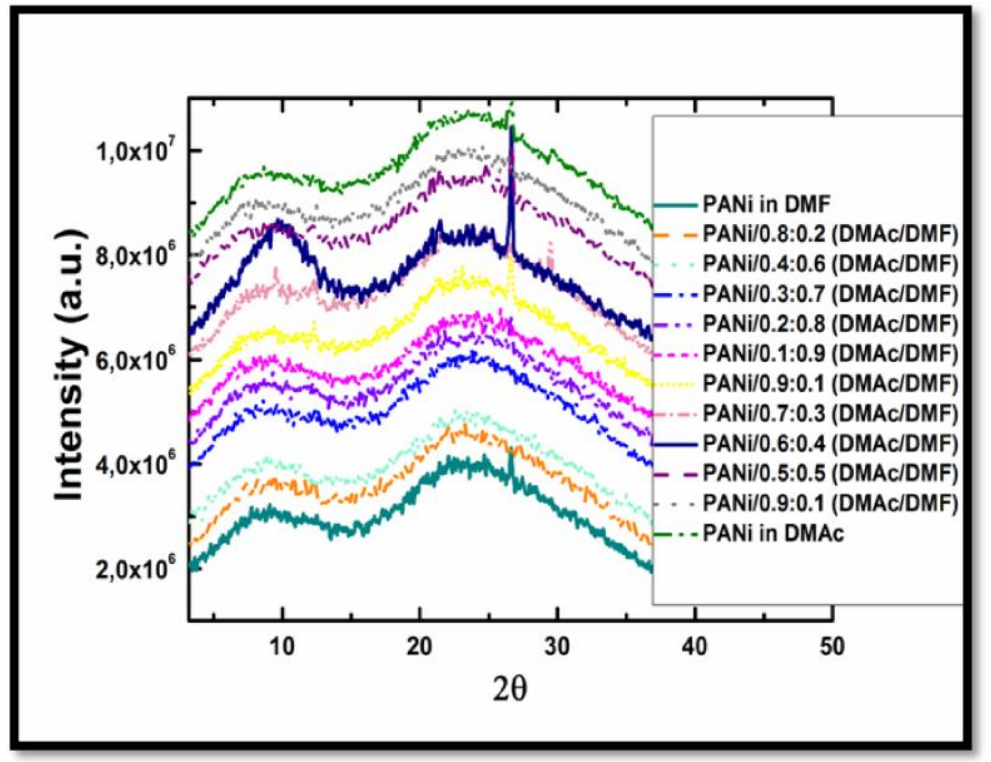

Figure 1. XRD patterns of the PANi films at different molar ratio of DMAc and DMF after heating treatment at $35^{\circ} \mathrm{C}$.

As is evident in PANI, broad diffraction peaks occur between $10^{\circ}$ and $30^{\circ}$ due to the parallel and perpendicular periodicity of the polymer (PANI) chain. The plot of PANi:0.5:0.5(DMAc/DMF) and
PANi :0.6:0.4(DMAc/DMF) in Fig.1, exhibited significant peak at diffraction angle of $27^{\circ}$ none of which are present in it PANi:0.4:0.6 (DMAc/DMF or PANi:0.8:0.2 (DMAc/DMF). The peak observed at 
$2 \theta \sim 30^{\circ}$ indicates the increase of crystalline structure in the PANi films ${ }^{23}$. T. Rajavardhana Rao et al. observed similar results for low molecular weight PVAEG and PVAEG-cu (II) systems. The diffraction peaks was sharp in $\mathrm{Cu}^{2+}$ complex of PVAEG films comparing with the pure PVAEG films and the crystalline phase increase after addition of $\mathrm{Cu}^{2+}$ ions ${ }^{24}$. In case of PANi:

0.6:0.4(DMAc/DMF), diffraction peaks are shifting towards higher angles from $2 \theta=9.7^{\circ}$ to $10.1^{\circ}$. The peaks are shifting toward higher angles indicate probably the formation of a stereo-complex between polymer and solvents on the glass substrates. The crystalline structure of this polymer may be regarded as an amorphous matrix in which small crystallites are randomly distributed.

Analysis of X-ray line profile is a simple and powerful method to estimate crystallite size. Among the available methods, Scherrer analysis is a simplified method where size average was estimated 25. The crystallite size D, which is about $13 \mathrm{~nm}$, was calculated from the Scherrer equation (Eq.1):

$$
\mathrm{D}=0.9 \lambda / \beta \cos \theta \quad \text { Eq.1 }
$$

Where $\lambda$ is the wavelength of the X-rays, $\theta$ is the diffraction angle, and $\beta$ is the corrected full width, at half-maximum of the peak. The estimation of the particle size is tested further by evaluating it with Meulenkamp equation ${ }^{26}$. According to Meulenkamp the wavelength $\lambda 1 / 2$ at which the absorption is $50 \%$ of that excitonic peak is directly related to the size of the particle via the fitted expression. The values of particle size evaluated from Meulenkamp equation is in fair agreement with the values obtained from Scherrer method Eq. 2.

$$
1240=\lambda_{1 / 2}\left(3.301+\frac{294}{D^{2}}+\frac{1.09}{D}\right)
$$

Eq. 2

\section{Raman spectra}

The Raman spectra of films prepared in the presence of DMAc/DMF reflect the differences in the molecular structure corresponding to polyaniline. The band corresponding to the $\mathrm{C}-\mathrm{C}$ stretching vibration of the benzenoid ring in the spectrum of the sample prepared in DMAc (Fig. 2-c) is shifted to $1680 \mathrm{~cm}^{-1}$ in comparison to the spectrum of a sample prepared in the presence of 0.6 DMAc: 0.4 DMF mixtures. Only a small shoulder at $1593 \mathrm{~cm}^{-1}$, assigned to the $\mathrm{C}=\mathrm{C}$ stretching of the quinoid ring vibrations ${ }^{27}$, is observed in the spectrum. The maximum of the band located at $1490 \mathrm{~cm}^{-1}$ and attributed to the $\mathrm{C}=\mathrm{N}$ stretching vibration in quinonoid units in the spectrum is detected. The maximum of the band attributable to $\mathrm{C}-\mathrm{N}^{+ \text {. }}$ vibrations is shifted from 1346 to $1338 \mathrm{~cm}^{-}$ ${ }^{1}$ and its intensity is lowered. The red shift of these bands corresponds most probably to the crosslinked and phenazine-like structures present in the sample. The position of the band at $1339 \mathrm{~cm}^{-1}$ reveals the presence of shorter and/or crosslinked structures or both. The peak of $\mathrm{C}-\mathrm{N}$ stretching vibrations of various benzenoid, quinonoid or polaronic forms observed at $1225 \mathrm{~cm}^{-1} \mathbf{2 8}$.

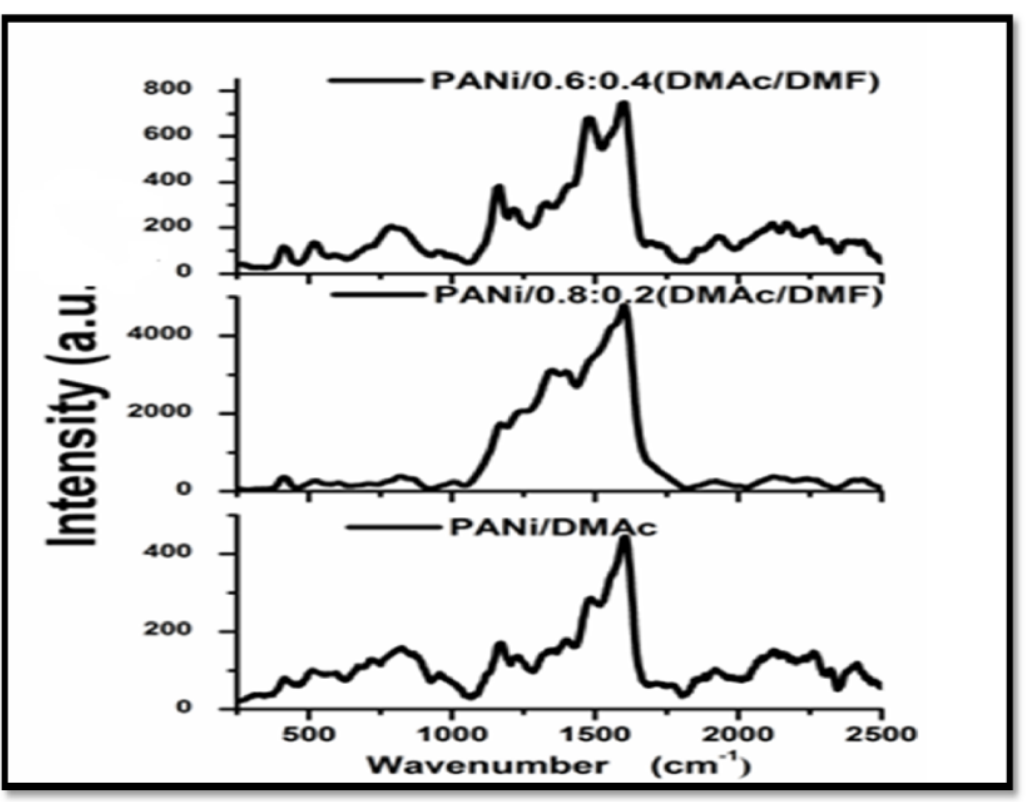

Figure 2. Resonance Raman spectra of polyaniline films: (a) PANi/0.6:0.4 (DMAc/DMF); (b) PANi/0.8:0.2 (DMAc/DMF) and (c) PANi/DMAc. $488 \mathrm{~nm}$. Baseline corrected.

The shape of the spectrum of PANi film prepared from a 0.6:0.4 molar ratio of DMAc and DMF (Fig. 2a) is similar to the spectrum of PANi film prepared in 0.5 DMAc/0.5 DMF mixtures. The two most intensive bands are situated at 1600 and $1505 \mathrm{~cm}^{-1}$. The two bands at 1262 and $1223 \mathrm{~cm}^{-1}$ of various $\mathrm{C}-\mathrm{N}$ stretching vibrations and the peak at $1171 \mathrm{~cm}^{-1}$ of $\mathrm{C}-\mathrm{H}$ bending vibration of the semiquinonoid rings are present in the spectrum. Bands at $815 \mathrm{~cm}^{-1}$ with a shoulder at $875 \mathrm{~cm}^{-1}$, attributed to benzene ring deformation ${ }^{29}$. The characteristic peaks at 1667 and $1033 \mathrm{~cm}^{-1}$ are attributed to the $\mathrm{N}-\mathrm{H}$ 
bending and $\mathrm{C}-\mathrm{N}$ stretching modes in the amines, respectively, which are due to the presence of stereo complex resulting in hydrogen bonding between DMAc or DMF molecules and amine $(\mathrm{NH})$ and imine $(=\mathrm{N})$ sites on the PANi chains. The changes in the spectra of the PANi base derived from the PANi film prepared from a 0.7:0.3 molar ratio of DMAc and DMF (Fig. 3a) are considerably smaller comparing to those of the PANi: 0.8:0.2 (DMAc/DMF). The decrease of the shoulder at 1610 $\mathrm{cm}^{-1}$ is observed. The band at $1496 \mathrm{~cm}^{-1}$ is shifted to $1480 \mathrm{~cm}^{-1}$ and its intensity increases. A small peak at $1379 \mathrm{~cm}^{-1}$ with a band at $1339 \mathrm{~cm}^{-1}$ corresponding to $\mathrm{C}-\mathrm{N}^{+}$. vibrations of charge delocalization, moved

to higher wavelength and decreased in intensity. A weak band at $1266 \mathrm{~cm}^{-1}$ are also present in the spectrum as well as the band at $1165 \mathrm{~cm}^{-1}$ of the $\mathrm{C}-\mathrm{H}$ bending vibration of quinonoid ring. In the region $\sim 1350 \mathrm{~cm}^{-1}$, the bands are related to the vibration of the protonated stretching vibration of the $\mathrm{C}-\mathrm{N}^{+}$. group and the changes in the intensities and positions of these bands reflect the conformational changes, leading to conversion to the PANi of the aromatic rings. The course of deprotonation is confirmed by the decrease in the intensity of the band at $809 \mathrm{~cm}^{-1}$. The decrease of the band at $1262 \mathrm{~cm}^{-1}$ can be linked to a decrease in the number of quinonoid and semi-quinonoid rings in the structure. The marked decrease of the band at $1170 \mathrm{~cm}^{-1}$, which is corresponding to the deformation vibration of the $\mathrm{C}-\mathrm{H}$ groups on the quinonoid rings, confirms the diminishing number of them ${ }^{28}$.

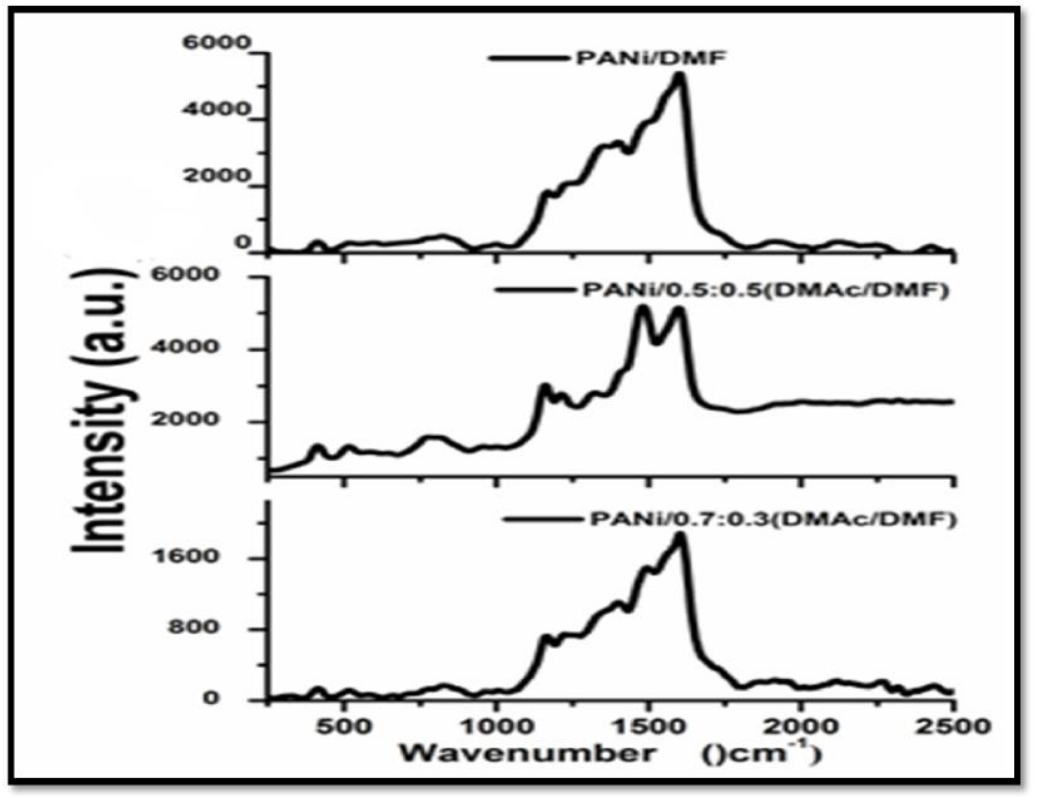

Figure 3. Resonance Raman spectra of polyaniline films: (a) PANi/DMF; (b) PANi/0.5:0.5 (DMAc/DMF) and (c) PANi/0.7:0.3 (DMAc/DMF). $488 \mathrm{~nm}$. Baseline corrected.

\section{Optical studies}

The energy gap defaults to the difference between the frontier orbitals, the HOMO and LUMO. In organic conjugated systems, HOMO is typically an occupied pi bonding orbital and LUMO is typically an unoccupied anti bonding orbital. Since an organic LUMO-HOMO excitation generates a highly bound exciton instead of free electron and a hole. The optical properties of the PANi films were investigated by optical transmittance and reflectance measurements. Figures 4-5 show the optical reflectance and transmittance spectra of PANi films .prepared at different molar ratio of compounds DMAc and DMF in the wavelength region from 300 to $2200 \mathrm{~nm}$. It can be observed that PANi films exhibit high transparency in near-infrared region. The average transmittance of films is over $80 \%$ in near-infrared region from 800 to $2200 \mathrm{~nm}$. One of the possible reasons for the transparency of films in the near-infrared could be the formation of a more planar conformation of polyaniline leading to favorable interaction between constituents. 


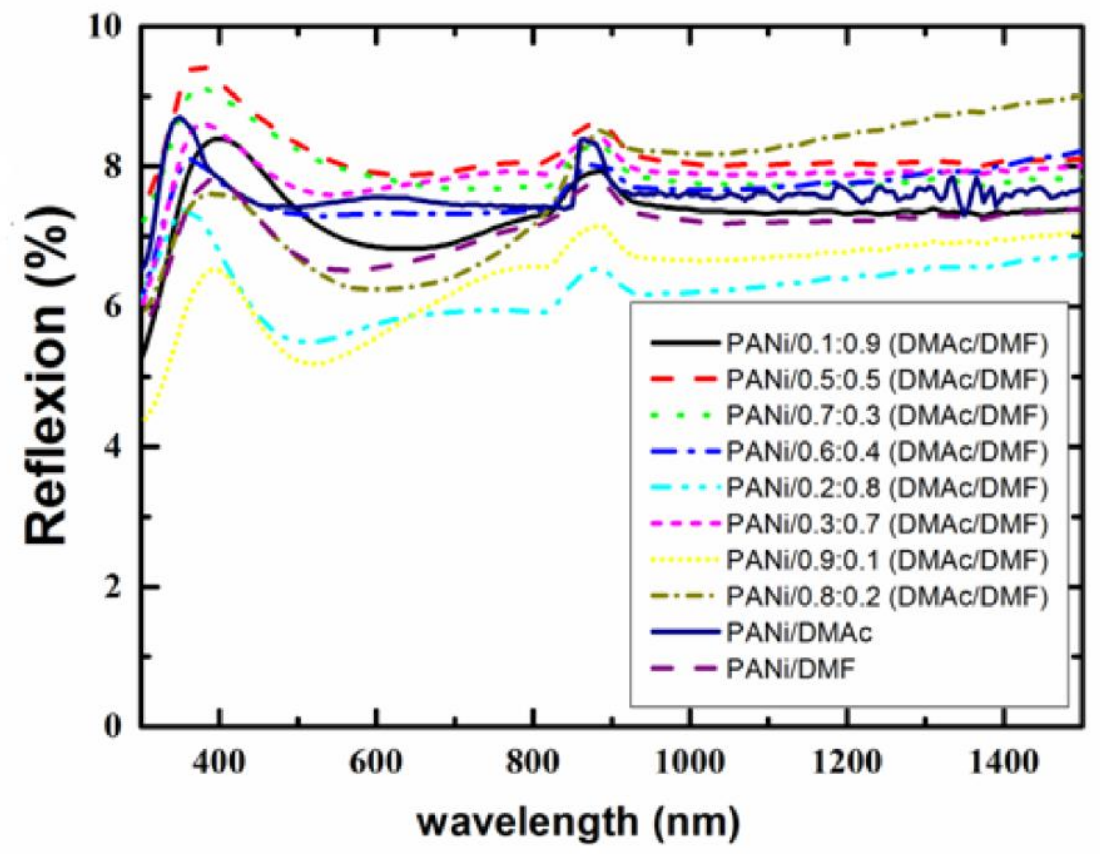

Fig.4: Optical reflectance spectra of films with the molar ratio of DMAc and DMF $=0.1: 0.9,0.2: 0.8,0.3: 0.7,0.5: 0.5,0.6: 0.4,0.7: 0.3,0.8: 0.2$ and 0.9:0.1 after heating treatment at $35^{\circ} \mathrm{C}$.

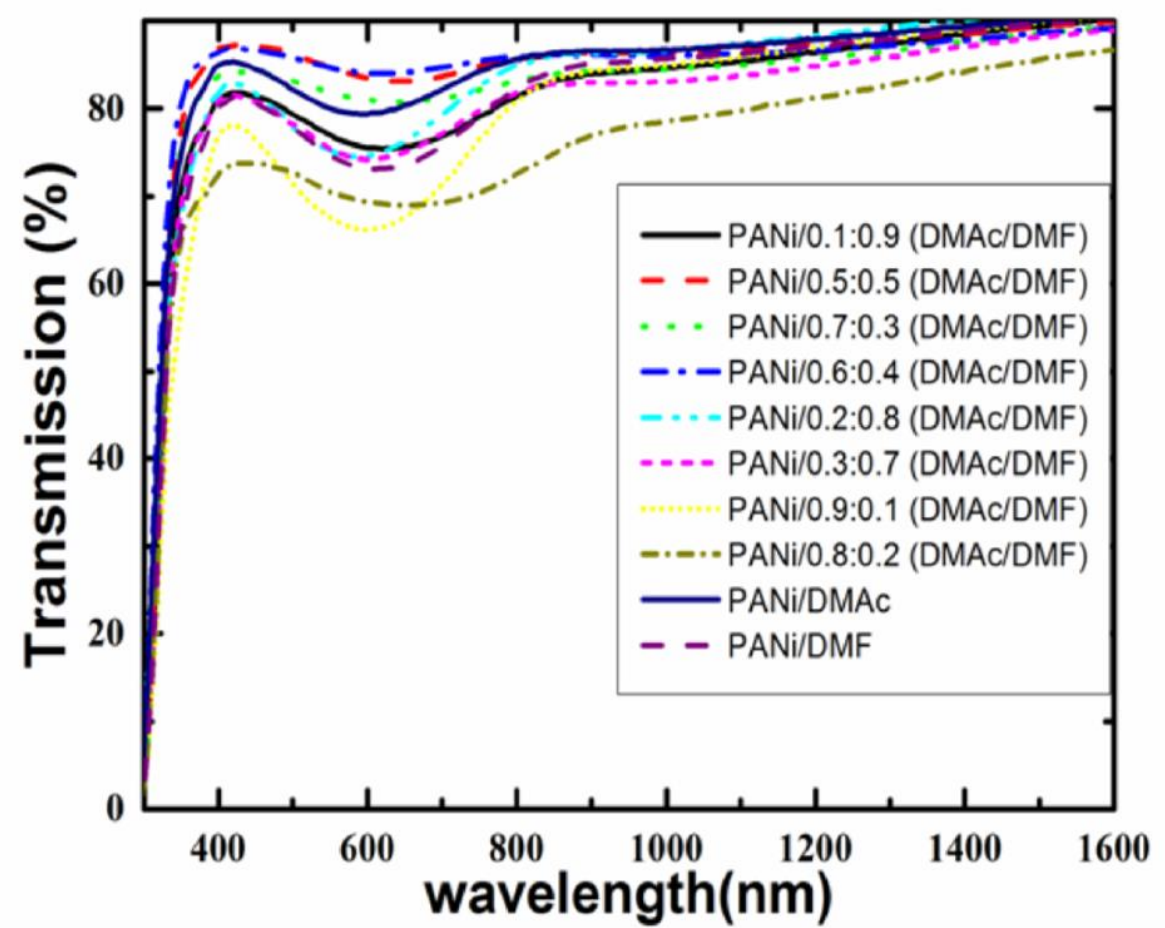

Figure 5. Optical transmittance of films with the molar ratio of DMAc and DMF = 0.1:0.9,0.2:0.8,0.3:0.7,0.5:0.5,0.6:0.4,0.7:0.3,0.8:0.2 and 0.9:0.1 after heating treatment at $35^{\circ} \mathrm{C}$

The study of the fundamental absorption edge in the UV region is a useful method for the investigation of optical transitions and electronic band structure in crystalline and non-crystalline materials. The optical absorption spectra can be used for the calculation of the value of energy band gap (Eg) for polymer sample.

The absorption coefficient $(\alpha)$ near the band edge varies with the photon energy $(h v)$ as the equation Eq.3:

$$
\alpha^{n}(E)=\beta\left(E-E_{g}\right)
$$

Eq. 3

Where $\mathrm{E}$ is the energy of the incidence photon, Eg is the optical band gap energy, $\beta$ is a constant known as the disorder parameter which is dependent on composition and independent to photon energy. Parameter $\mathrm{n}$ is the power coefficient with the value that is determined by the type of possible electronic transitions, i.e.n=1/2, 3/2, 2 or $1 / 3$ for direct allowed, 
direct forbidden, indirect allowed and indirect forbidden respectively. The direct band gap energy can be determined from the extrapolation of the linear section of the curves to $\mathrm{x}$-axis, as described by Mott \& Davis concept. The optical band gaps of the PANi films obtained from Figure 6-8 are around $3.57 \mathrm{eV}$. Thamilselvan et al. reported optical and magnetic characterizations of the interaction between polyaniline and copper oxide $\mathrm{Cu}_{2} \mathrm{O} / \mathrm{PANI}$ Nanocomposite ${ }^{30}$. In their opinion, the increase of

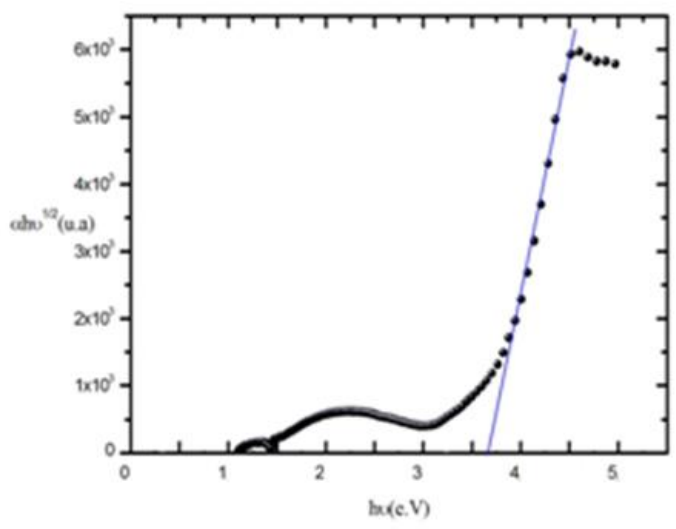

(a) band gap of $\mathrm{Cu}_{2} \mathrm{O}$ compared with its bulk band due to the presence of bioorganic phases on $\mathrm{Cu}_{2} \mathrm{O}$ due to the formation of polaron and bipolaron charge carrier in the nanocomposite. The substitutions in the ring affect the tensional angle; hence dihedral/torsional angle between adjacent aromatic rings is increased 31. It caused the increase in twisting of torsional angle which increases the band gap as compared with the band gap of polyaniline.

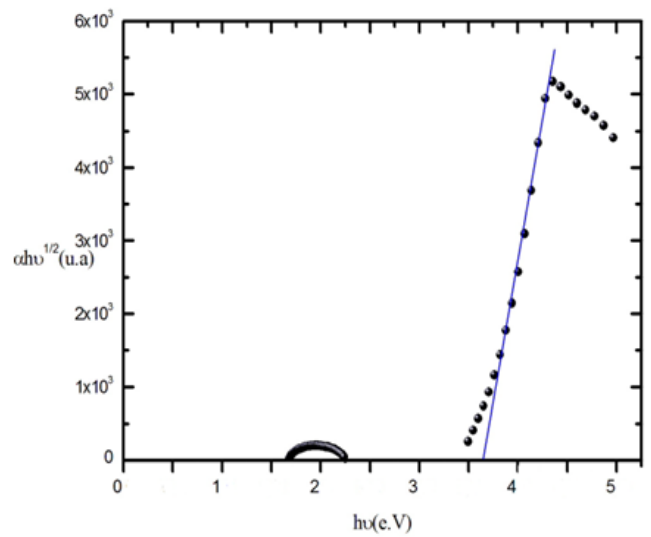

(b)

Figure 6. Plot of $(\alpha h v)^{1 / 2}$ versus photon energy (hv) of the PANi films: (a) PANi/DMAc and (b) PANi/DMF

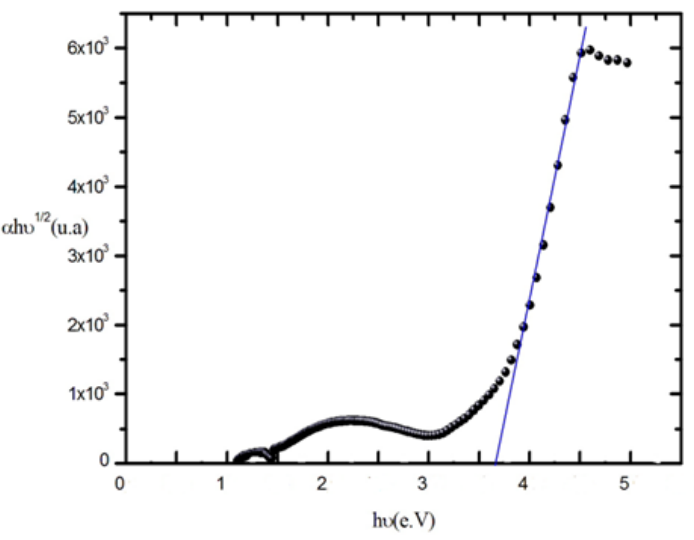

(c)

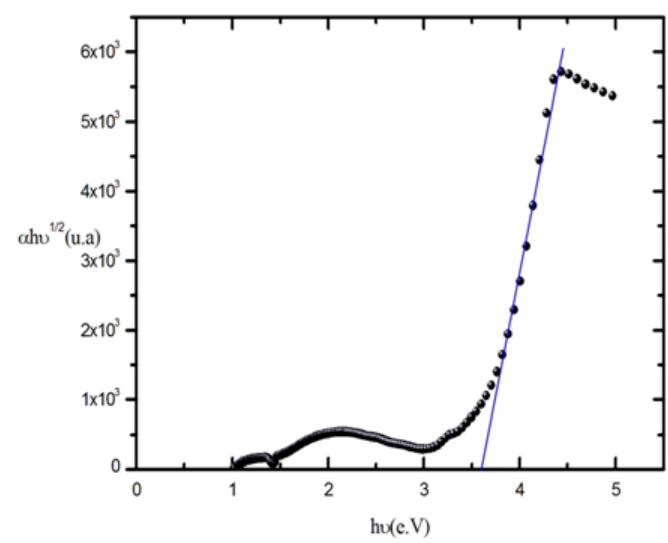

(d)

Figure 7. Plot of $(\alpha h v)^{1 / 2}$ versus photon energy (hv) of the PANi films: (c) PANi/0.5:0.5 (DMAc/DMF) and (d) PANi/0.6:0.4 (DMAc/DMF).

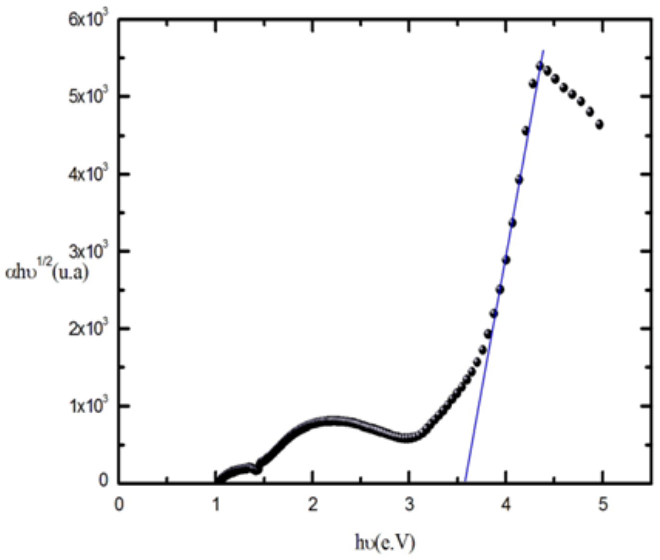

(e)

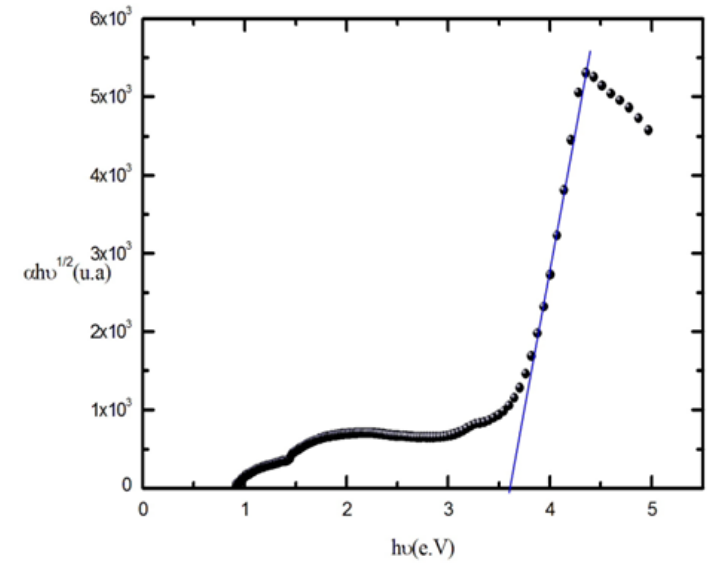

(f)

Figure 8. Plot of $(\alpha h v)^{1 / 2}$ versus photon energy $(h v)$ of the PANi films: (e) PANi/0.7:0.3 (DMAc/DMF) and (f) PANi/0.8:0.2 (DMAc/DMF). 


\section{Fluorescence spectral}

Figure 9 illustrates the photoluminescence (PL) spectra obtained from the PANi films. Its PL increase with a change of DMAc/DMF contents. Musa et al. showed that the PANi polymer had two broader emission peaks with wavelength at 420 and $470 \mathrm{~nm}$ respectively for $\mathrm{EB}$ and $\mathrm{ES}^{32}$.

PL spectra of PANi: DMAc/DMF cast films of a series of molar ratio (0.7:0.3; 0.8:0.2 and 0.9:0.1) were investigated under laser excitation around 300 $\mathrm{nm}$. The PL peaks of $480 \mathrm{~nm}$ are broadened and shifted to lower wavelength $(\sim 380 \mathrm{~nm})$. The PL of PANi: 0.6:0.4 (DMAc/DMF and PANi: 0.5:0.5 (DMAc/DMF) thin films exhibited two peaks at 420 and $480 \mathrm{~nm}$. The PANi: 0.6:0.4 (DMAc/DMF) film has a somewhat stronger peak at $480 \mathrm{~nm}$ that of the PANi: 0.3:0.7 (DMAc/DMF) or PANi: 0.5:0.5 (DMAc/DMF) films. The thin film with polymer cast from 0.6:0.4 and 0.5:0.5 molar ratio of (DMAc/DMF), exhibited the highest PL increase without any peak shift. We suggest the difference in PL peak position for polymer cast films should be attributed to the different dispersion states of solvent molecules in polymer matrices due to the differences in the interaction and miscibility between DMAc/DMF and each polymer. PANi appeared to be well dispersed in 0.6:0.4(DMAc/DMF) solvents but some particles of aggregated polymer molecules are formed in PANi :0.7:0.3 or PANi: 0.8:0.2 thin film. We refer to the earlier reports of Zhang et al. where PL increases with an increase of rare earth ions ${ }^{33}$. The emission peaks of polyaniline doped with $\mathrm{Fe}^{3+}$ and $\mathrm{Al}^{3+}$ ions at the excitation wavelength of $370 \mathrm{~nm}$ are located at $477 \mathrm{~nm}$. The Fe-PANi or AL-PANi exhibited a very weak PL peak around 410 $\mathrm{nm}$, while their main emission peak shifted to higher wavelength with fluorescent intensity increase. In their opinion, the progressive increase of fluorescent intensities led eventually to the complexation between $\mathrm{Fe}^{3+}$ and $\mathrm{Al}^{3+}$ ion with the nitrogen atoms in the polymeric chains.

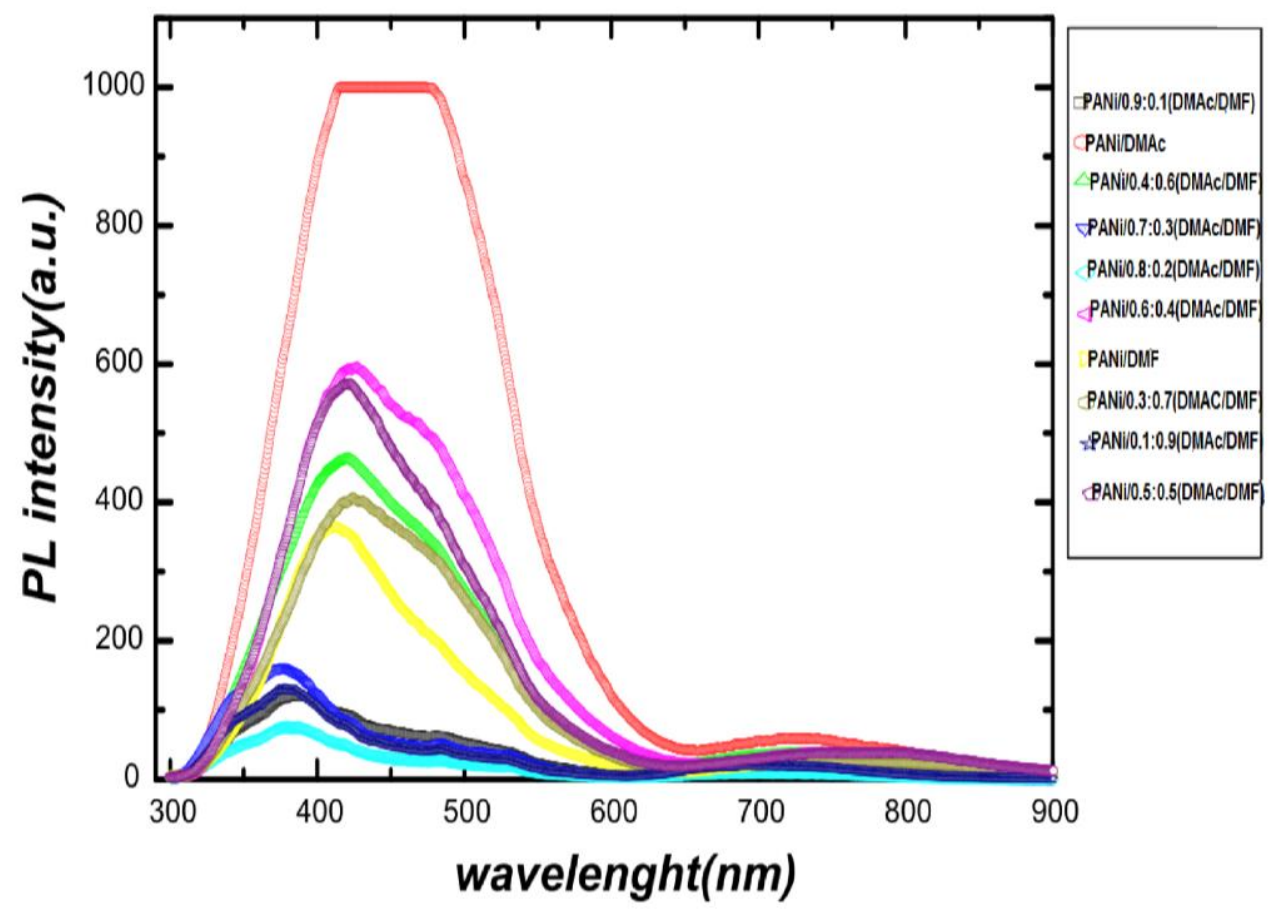

Figure 9. Photoluminescence spectra of the PANi films, under $300 \mathrm{~nm}$ excitation.

The same observation was also made for the PANi films. An increase in the rigidity of its structure observed an increase in the fluorescence intensity .Since formation of complex PANi/0.6(DMAc/0.4(DMF) has already contributed towards the rigidity of the structure. With the

\section{SEM studies}

Morphologies of the PANi films with different decreasing of fluorescent intensity (as shown in Figure 9), a cross-linking structure is eventually formed can be explained by the formation of crosslinking structure by 0.8 DMAc/0.2 DMF or 0.7DMAc/0.3 DMF interaction with adjacent polyaniline chains.

DMAc/DMF contents are shown in Fig. 10-12. 


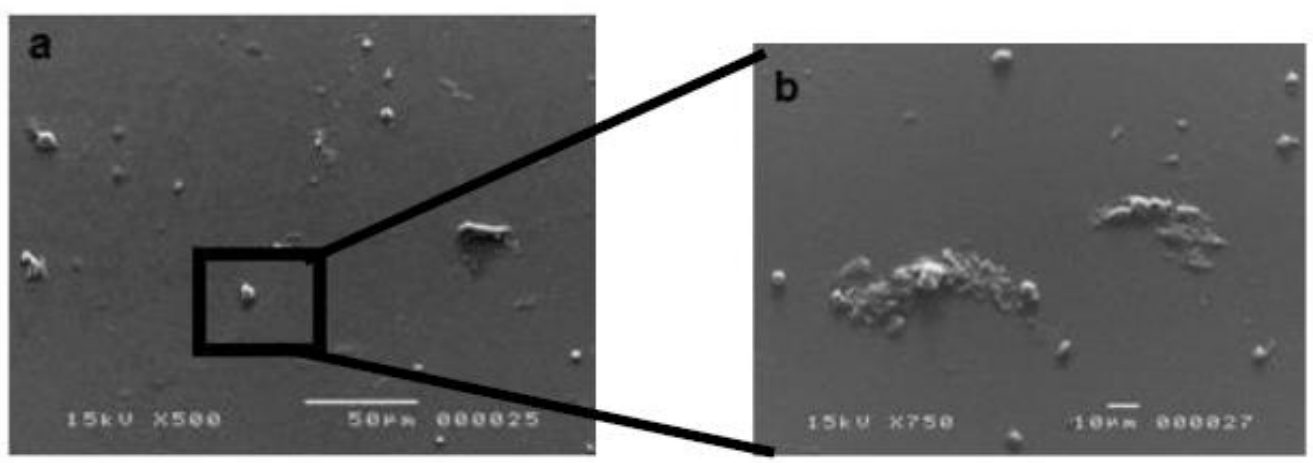

Figure 10. SEM image of PANi /0.6:0.4 (DMAc/DMF) film

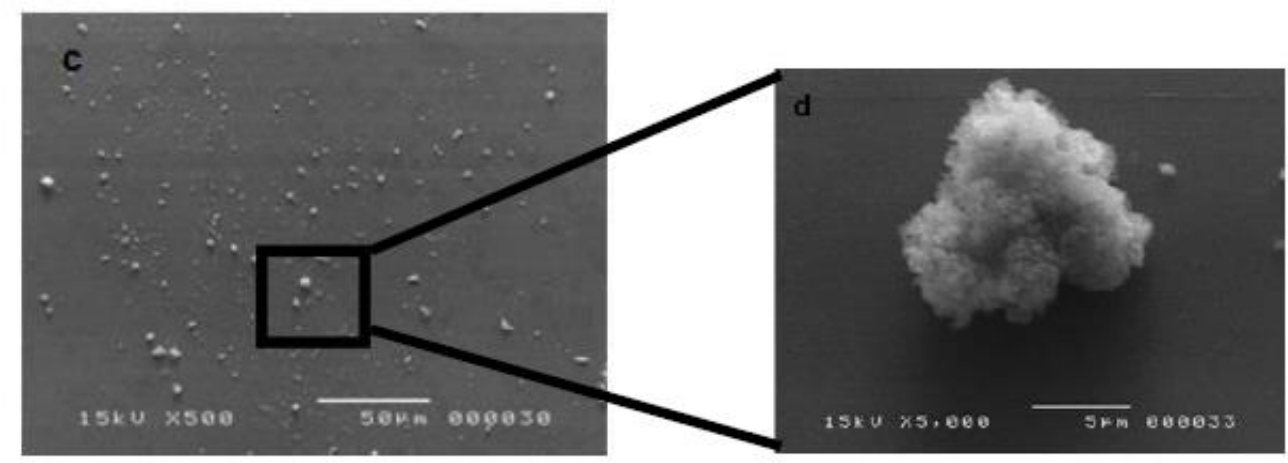

Figure 11. SEM image of PANi / 0.8:0.2 (DMAc/DMF) film

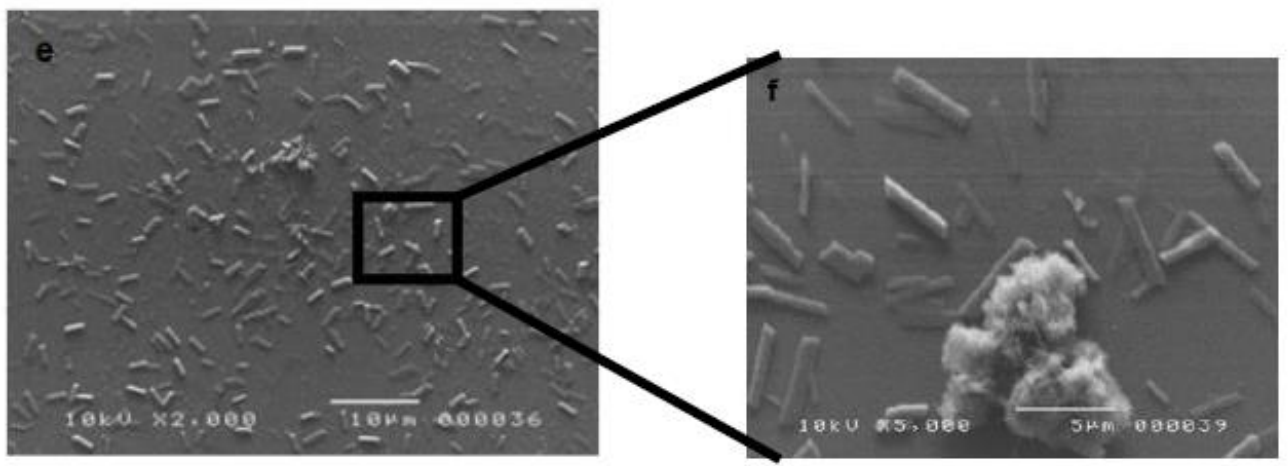

Figure 12. SEM image of PANi/0.7:0.3 (DMAc/DMF) film

All the studied systems reveal flaky shaped structure, in which the size of flakes increases as certain DMAc/DMF is added into PANi. It can be seen that the size of final cluster of DMAc/DMF covered by PANi with which 0.8:0.2 (DMAc/DMF) nanorods content due to the possibility of agglomeration of particles, and PANI has declined.The SEM images help us draw a conclusion that the DMAc/DMF casting solvent has a strong effect on the morphology of PANi, since PANi has various structures such as granules, nanofibers, nanotubes, nano- spheres, microspheres and flakes ${ }^{34}$. According to the SEM micrographs in Fig.10, PANi and 6DMAc/4DMF crystal have formed a nanocomposite in which the nanorods are embedded in the polymer matrix.

\section{Conclusion}

The polyaniline cast films have been prepared in DMAc/DMF solutions. Structural analysis indicated that the interactions of PANi with DMAc/DMF solvents occur at the nitrogen atom of imine groups on polymeric chain backbones. Two DMAc/DMF compositions can significantly increase the fluorescent intensity of polyaniline due to a steady plane rigid structure for PANi chains formed by the complex of nitrogen atoms on polymer chains with DMAc or DMF molecules. The result indicates that DMAc/DMF mixtures can sensitize the fluorescence of PANi by improving the structure of polymeric chains. Addition of DMAc/DMF into polyaniline matrix increases the direct band gap $(3.57 \mathrm{eV})$ of the films when compared to polyaniline. 
This gap increases with increases the highlight of this work is the simple production of highly transparent thin films with electrical and optical properties appropriate for optoelectronic applications.

\section{References}

1- A.G. MacDiarmid, Angew. Chem. Int.2001, 40, 2581-2590.

2- C.D. Dimitrakopoulos, P.R.L. Malenfant, Adv. Mater. 2002, 14, 99-117.

3- S.Virji, R.B. Kaner, B.H. Weiller, Chem. Mater. 2005, 17, 1256-1260.

4- S. Venkatachalam, P.V. Prabhakaran, Synth .Met. 1998, 97, 141-146.

5- Z.M.Tahir, E.C. Alocilja, and D.L. Grooms, Sensor. 2007, 7, 1123-1140.

6- L. Liang, J. Liu, C.F. Windisch, G.J. Exarhos, and Y. Lin, Angew.Chem. Int. 2002, 41, 3665-3668.

7- L. F. He, Y. Jia, F. L. Meng, M. Q. Li, and J. H. Liu, Mater. Sci. Eng. 2009, 163, 76-81.

8- Y. H. Lin, X. L. Cui, Chem. Commun. 2005, 2226-2228.

9- M. Q. Li, L. H. Jing, Electrochim. Acta. 2007, 52, 3250-3257.

10- V. Mottaghitalab, B. B. Xi, G. M. Spinks, and G. G. Wallace, Synth.Met. 2006, 156, 796-803.

11- G. M. Spinks, V. Mottaghitalab, M. BahramiSaniani, P. G. Whitten, and G. G. Wallace, Adv. Mater. 2006, 18, 637-640.

12- S. Ben-Valid, H. Dumortier, M. Decossas, R. Sfez, M. Meneghetti, A. Bianco, and S. Yitzchaik, J. Mater. Chem. 2010, 20, 24082417.

13- P. C. Ramamurthy, A. M. Malshe, W. R. Harrell, R. V. Gregory, K. McGuire, and A. M. Rao, Solid State. Elect. 2004, 48, 2019-2024.

14- J. H. Lim, N. Phiboolsirichit, S. Mubeen, M. A. Deshusses, A. Mulchandani, and N. V. Myung, Nanotech. 2010, 21, 075502.

15- B. L. He, B. Dong, W. Wang, and H. L. Li, Mater. Chem. Phys. 2009, 114, 371-375.

16- S. R. Sivakkumar, W. J. Kim, J.A. Choi, D. R. MacFarlane, M. Forsyth, and D.W. Kim, J.Power. So. 2007, 171, 1062-1068.

17- S. Bhadra, D. Khastgir, N. Singha and J. Lee, Progress in Polym. Sci. 2009, 34, 783-810.
18- S.G. Oh, B.J. Kim, M.G. Han, and S.S. Im, Synth. Met. 2001, 122, 297-304.

19- X.G. Li, M.R. Huanga, J.F. Zeng, and M.F. Zhu, Colloids and Surfaces A: Physicochem. Eng. 2004, 248, 111-120.

20- R. Sainz, A. M. Benito, M. T. Martinez, J. F. Galindo, J. Sotres, A. M. Baro, B. Corraze, O. Chauvet, A. B. Dalton, R. H. Baughman, and W. K. Maser, Nanotech. 2005, 16, 150154.

21- Y. F. Ma, S. R. Ali, L. Wang, P. L. Chiu, R. Mendelsohn and H.X. He, J. American Chem. Soc. 2006, 128, 12064-12065.

22- N. Bohli, F. Gmati, A. B. Mohamed, V. Vigneras and J. L. Miane, J. Phys. D: Appl. Phys. 2009, 42, 205404.

23- D. Prasanna, H. S. Jayanna, A.R. Lamani, M. L. Dinesha, C. S Naveen, and C. Shankaramurthy, J. Chin. Phys. Lett. 2011, 28, 7701.

24- T. Rajavardhana Rao, I. Omkaram, K-V. Brahmam, Ch. Linga Raju, J. Mol. Struct. 2013, 1036, 94-101

25- H. Klug, L. Alexander, Eds.; X-ray Diffraction Procedures, Wiley: New York, 1962, pp.125.

26- E. A. Meulenkamp, J.Phys. Chem. B. 1998, 102, 5566-5572.

27- M. Cochet, G. Louarn, S. Quillard, M-I. Boyer, J-P. Buisson, S. Lefrant, J. Raman Spectrosc. 2001, 11, 1029-39.

28- I. Sedenkova, M. Trchova, J. Stejskal, Polym. Degrad. Stability, 2008, 93 , 2147-2157

29- A. Uygun, L. Oksuz, A. G. Yavuz, A. Guleç, and S. Sen , Current App.Phys. 2011, 11, 250-254.

30- K. Gopalakrishnan, C. Ramesh, M. Elango, and M. Thamilselvan, Mat. Sci. 2014, ID 567927.

31- V. A. Khati, S. B. Kondawar, and V. A. Tabhane, Analytical \& Bioanalytica Electrochem. 2011, 3, 614-624.

32- F. S. Mehamod, R. Daik and A. Musa, Malaysia. J. Chem. 2002, 4, 35-40.

33- Z. Jiali, W. Hao, Y. Shimei, W. Shaohui, Y. Shaoming, J. App. Polym. Sci. 2012, 125, 2494-2501.

34- J .Stejskal, I .Sapurina, M .Trchova, Progress In Polym. Sci, 2010, 35, 1420-1481. 